\title{
El aprendizaje comprensivo como soporte de la formación básica en matemáticas. Una aproximación desde el trabajo investigativo en educación matemática
}

\author{
Edgar Alberto Barón Poveda ${ }^{\star}$, Hugo Edver Zamora Coronado ${ }^{\star \star}$ \\ Politécnico Grancolombiano
}

FECHA DE RECEPCIÓN: NOVIEMBRE 1 DE 2010

FECHA DE APROBACIÓN: FEBRERo 20 DE 2011

\begin{abstract}
Resumen Las tensiones entre requerimientos sociales respecto de una formación básica en matemáticas para un profesional en ciernes y los imaginarios colectivos construidos alrededor de dicha formación, requieren de reflexión y propuestas que acerquen las posiciones con objeto de contribuir desde la educación superior a una formación de calidad. En este artículo, proponemos dos partes. En la primera, presentamos una reflexión acerca de cómo es entendida la escolarización del conocimiento matemático desde la enseñanza y cómo desde el aprendizaje y en la segunda, un acercamiento a lo que ha significado para el grupo de investigación comenzar a trabajar en lo disciplinar con una mirada histórica, epistemológica para construir una propuesta de reorientación de la actividad escolar en matemáticas que se fundamente en el aprendizaje comprensivo de nociones y conceptos, específicamente en ecuaciones.
\end{abstract}

\begin{abstract}
The tensions among social requirements with respect to a basic training in mathematics for a potential professional and the collective imagination built around such training require reflection and proposals that bring together the different positions in order to contribute from the higher education to have quality training. In this article, two parts are proposed. The first one is a reflection about the way the education of mathematical knowledge is understood from the teaching and from the learning position. The second one is an approach to the meaning that working within the disciplinary field has for the research group with a historical, and an epistemological point of view in order to create a
\end{abstract}

* CMg Educación. Licenciado en Matemáticas. Profesor de planta del Politécnico Grancolombiano. Entre sus intereses académicos se encuentran la educación matemática, el problema de la elección colectiva y el bienestar social, y la topología. eabaron@poli.edu.co

** CMg Educación. Especialista en Educación a Distancia. Profesor de planta del Politécnico Grancolombiano. Entre sus intereses académicos se encuentran la educación matemática y la formación de docentes de matemáticas en educación básica. hzamora@poli.edu.co 
reorientation proposal regarding school activities in mathematics based on the comprehensive learning of notions and concepts, specifically equations.

Palabras Clave: escolarización del conocimiento matemático, enseñanza, aprendizaje, red de análisis didáctico, ecuación.

Keywords: education of mathematical knowledge, teaching, learning, didactic analysis network, equation.

\section{Parte I}

\section{Sistema escolar y construcción de imaginarios colectivos}

Desde diferentes entornos sociales se han formulado interrogantes acerca de las características de las experiencias escolares con el conocimiento matemático, y de las estrategias que se proponen para posibilitar que las actividades escolares con base en el conocimiento matemático contribuyan al desarrollo del pensamiento necesario para que un individuo ejerza en el mundo que le espera. Las respuestas han sido de diverso orden, desde los intentos individuales por mejorar la práctica docente, pasando por la conformación de amplios programas de investigación que apuntan a la reflexión, indagación y proposición de soluciones a las problemáticas derivadas de la escolarización del conocimiento matemático, y culminando en las reformas escolares que expresan la voluntad de acercar la escolaridad a las exigencias sociales. Sin embargo, una afirmación desde diferentes voces sociales ronda en forma permanente estos esfuerzos: los aprendizajes en matemáticas logrados por los egresados del sistema escolar son mecánicos y no corresponden a los requerimientos del entorno donde ejerce el nuevo profesional.

Es pertinente entonces la mirada a la responsabilidad que compete a los diferentes estamentos del sistema escolar, respecto de la afirmación dada, con el propósito de reflexionar y actuar en pos del logro en los estudiantes de aprendizajes comprensivos en matemáticas. En particular, el reconocimiento y estudio de los supuestos que orientan la acción escolar en torno a las matemáticas, en los ambientes de educación superior, es requisito esencial para la reflexión y para el diseño de propuestas que, por un lado, repercutan en la decisión de un egresado respecto a su elección de carrera profesional y, por otro lado, en la calidad de formación del egresado de la universidad.

Los procesos que relacionan el conocimiento matemático escolar, con la formación básica de un estudiante que accede a la educación superior, están sometidos a tensiones propias de las exigencias sociales, los supuestos del sistema escolar y las creencias de los actores involucrados en dichos procesos.

El sistema escolar asume en general que la secuencialidad en los procesos educativos entrega a la universidad un estudiante con herramientas intelectuales básicas de desempeño académico y, en particular, con un bagaje de conocimiento matemático escolar que le habilita para comenzar trabajos alrededor de la estructuración de un pensamiento que le permita hacer abstracciones y dar explicaciones desde lo disciplinar a sus procesos de elaboración de conocimiento matemático. 
El estudiante que comienza su proceso de formación profesional, parte del hecho de que su fundamentación en matemáticas corresponde a lo que le fue enseñado y tiene una mirada acerca de las matemáticas de tipo mecánico, que además no siempre puede replicar correctamente en situaciones similares a las enseñadas. De alguna manera, funda su accionar en la creencia que tanto el sistema escolar como la sociedad conocen las fortalezas y debilidades de los procesos en la educación básica y que por lo tanto los vacíos que tiene en cuanto a dominio de nociones y conceptos en matemáticas, serán suplidos por los planes de estudio de la educación superior. Además cree firmemente que la responsabilidad con el conocimiento matemático escolar es propiedad del sistema escolar y en particular de su representante visible: el docente, pues el maestro es quien sabe y por tanto a quien hay que entregar cuentas y quien juzga el desempeño estudiantil.

El maestro de matemáticas, punto de encuentro de intereses sociales, escolares y estudiantiles, soporta su práctica docente en creencias vinculadas usualmente a visiones como:

1. El estudio profundo del conocimiento matemático inmerso en nociones y conceptos que se desarrollan en matemáticas escolares es suficiente y necesario para garantizar un ejercicio docente que contribuya a la formación básica del estudiante. "De la necesidad no hay duda, no así de la suficiencia" 7].

2. Es responsabilidad del estudiante identificar herramientas intelectuales que le aproximen al conocimiento propuesto en el aula de clase y logre por tanto dominios similares a los que muestra el docente cuando enseña.

3. Las herramientas de la pedagogía son mediadores entre el ejercicio docente y la responsabilidad que tiene el estudiante frente a los procesos de aprendizaje y que contribuyen a acortar distancias en visiones sobre el trabajo con matemáticas escolares, sin exonerar a estudiantes y maestros del papel que les corresponde en el proceso.

Las dinámicas impuestas por las tensiones brevemente descritas se configuran en un círculo de responsabilidades frente al conocimiento matemático escolar: la sociedad responsabiliza al sistema escolar, éste responsabiliza al docente, el docente responsabiliza al estudiante quien devuelve la responsabilidad al representante de la sociedad y el sistema escolar: el docente. En esta dinámica de ires y venires, se ha abandonado al elemento central del proceso: el aprendizaje comprensivo de nociones y conceptos en matemáticas.

El conocimiento matemático es concebido como un conocimiento terminado y por tanto elaborado por otros, de tal forma que los procesos escolares se entienden como caminos que permiten acceso a los resultados vinculados con algoritmos, fórmulas o procedimientos donde actúan nociones y conceptos, éstos últimos relegados en su importancia frente a la aplicación de algoritmos.

Con el objeto de conciliar exigencias, supuestos y creencias en torno al aprendizaje en entornos escolares, sobre nociones y conceptos de matemáticas, es preciso repensar los roles de los actores del sistema escolar frente al conocimiento matemático escolar. Las visiones que se construyan en este sentido deben redefinir los compromisos con el conocimiento e identificar los elementos centrales que 
posibiliten que el aprendizaje comprensivo de nociones y conceptos sea el núcleo central de la actividad de docentes, estudiantes y demás entes que intervienen en el acto educativo.

\section{Concepción de la función de la escolaridad en dos perspectivas}

Los procesos de identificación y descripción de nuevos roles dentro del sistema escolar en la perspectiva enunciada atienden a una gran complejidad, la cual debe ser materia de investigación amplia y profunda. Aquí se plantea una observación acerca de un aspecto de esta problemática: la concepción de escolaridad.

Es de anotar el esfuerzo de la comunidad educativa global por tratar de caracterizar una escolaridad que refleje las exigencias sociales. En "La educación encierra un tesoro", Jacques Delors delinea los cuatro pilares sobre los cuales debería soportarse la educación en el siglo XXI y hacia los cuales debería enfocarse la acción de los niveles de escolaridad para que sean asumidos por un niño o adolescente en proceso de formación ciudadana. Aprender a conocer, aprender a hacer, aprender a ser y aprender a vivir juntos constituyen la utopía de los quehaceres y metas de la escolaridad formal o informal en la visión de Delors. Este derrotero marca expectativas de reflexión y acción, sobre el papel a desempeñar por la institución escolar y por sus actores: estudiantes, maestros, administradores escolares y currículos en la concreción de propósitos y fines de la educación. En especial el ítem que señala un requerimiento sobre el aprender a conocer intenta orientar los procesos escolares hacia la consecución de aprendizajes que promuevan la autonomía intelectual y desencadenen en el individuo procesos que lo lleven a niveles de comprensión cada vez mejores.

Para el ente universitario se señala implícitamente la tarea de contribuir solidariamente con el desarrollo del estudiante, especialmente con un pensamiento superior, pero también se le exige que asuma con responsabilidad el real estado intelectual del individuo respecto de los supuestos y exigencias para abordar dichas tareas. Por lo tanto, se sitúa la función de docencia de la educación superior en un plano que compromete seriamente los planteamientos institucionales, en cuanto no es suficiente insertar en los planes de estudio unas cuantas asignaturas de matemáticas, sino que es imprescindible la declaratoria del tipo de formación básica en matemáticas que se persigue con ellas y de las formas como se propone la nivelación del estudiante para lograr alcanzar los mínimos requeridos por tal tipo de formación.

Desarrollar planes, en coherencia con nuevas visiones del papel de la docencia universitaria en matemáticas, significa el reconocimiento de las posibilidades de "unas matemáticas para todos", entendida esta expresión en términos de la real posibilidad que se da a quienes ingresan a las aulas universitarias, de vivenciar la experiencia escolar matemática y de dotarla de sentido en el marco de su formación profesional.

Ahora, el desarrollo de los planes antes referidos requiere de la reflexión que supere la consideración metodológica o la técnica como el soporte de su diseño. 
En este sentido es necesaria la mirada a la función asignada por la sociedad a la escolaridad para caracterizarla e identificar la viabilidad de estrategias y planes que replanteen la función de docencia.

La investigación en educación matemática ha identificado la enseñanza como la función esencial de la escolaridad. La función de "enseñar" ha sido caracterizada en elementos fundamentales y en relaciones entre ellos, que para el caso de las matemáticas, se pueden describir a la manera de Ortíz [5], en este caso, sin profundizar la reflexión en ninguno de ellos:

- Maestros de matemáticas formados para que cumplan la función de enseñar transmitiendo conocimientos, y evaluar a sus alumnos según los resultados obtenidos a partir de la enseñanza.

- Conocimientos matemáticos elaborados por otros, que por razones no explícitas se convierten en conocimientos escolares o contenidos a enseñar.

- Alumnos que aprenden, en general, por repetición de datos que no alcanzan a ser información y mucho menos conocimiento.

- Enseñanza de conocimientos matemáticos que una vez adquiridos por el alumno se supone deben ser aplicados por transferencia a los contextos en que se requieran. Esta enseñanza se enmarca en valores como la meritocracia; trato igual para todos los alumnos, a todos se les dan las mismas posibilidades y se les plantean las mismas exigencias; méritos individuales justifican la desigualdad de los resultados, y la evaluación como mecanismo de selección, aplicado al final de un período lectivo. El maestro a través de la evaluación califica o descalifica a los alumnos por sus logros frente a lo enseñado.

- Enseñanza que tiene como meta la transferencia y aplicación eficiente de conocimientos, se enmarca en un por qué y para qué no claramente explicitados, con los cuales, alumnos y maestros tienen poca o ninguna identificación, es decir, maestros y estudiantes que no se hacen responsables del aprendizaje. Para unos y otros siempre hay causas ajenas que justifican los resultados deficientes.

La enseñanza tradicional se soporta en la concepción filosófica del conocimiento expuesto desde diferentes corrientes del positivismo, la cual asume el conocimiento como objeto de transmisión, más que como objeto de construcción. Los conocimientos enseñados se aceptan como verdaderos, inmodificables, correspondientes con la realidad e independientes del tiempo, el espacio y los individuos que enseñan y aprenden.

Las exigencias sociales a la escolaridad, en los términos de nuevos requerimientos intelectuales a sus egresados sitúan teóricamente al aprendizaje como el eje central de su razón de ser. Con una nueva función para la institución educativa, es necesario redefinir los elementos que intervienen en el aprendizaje y lo determinan, así como las relaciones entre éstos. En esencia son los mismos que constituyen la enseñanza, pero con características y relaciones diferentes, pues se soportan en una concepción epistemológica opuesta a la tradicional. Tales elementos han sido caracterizados así [5]:

- Maestros de matemáticas formados para orientar el aprendizaje. Profesionales de la docencia, trabajadores intelectuales, que viven y propician una cultura 
académica. Maestros comprometidos con el aprendizaje y que responden solidariamente por él.

- Conocimientos matemáticos escolares contextualizados que llegan a ser tales, porque a través de su proceso de difusión y aplicación o histórico de construcción mostraron ser, y aún son válidos y necesarios para la vida, la ciencia y la tecnología, convirtiéndose en lo que hemos denominado "conocimientos socialmente aceptados y exigidos" y por tanto necesarios de aprender.

- Estudiantes que asumen conscientemente y con responsabilidad la tarea de construcción de sus conocimientos y, por ende, la modificación y construcción de sus estructuras mentales. La transformación intelectual es una decisión del estudiante y, por tanto, un acto de la razón.

- Aprendizaje que en contraste con la enseñanza es particular, depende de las experiencias individuales, las capacidades, posibilidades y limitaciones del estudiante y su entorno que sólo se da en la medida que se posibilite y genere en conocimientos anteriores y estructuras de pensamiento logradas, y se obtiene a través de procesos de construcción basados en la actividad individual, colectiva y por la confrontación social.

- Evaluación autocrítica permanente que revisa el proceso de construcción en que están comprometidos estudiantes y maestros y permite identificar oportunamente: lo realizado y el cómo se realizó; los logros y lo que aún no se puede considerar como tal porque no se ha comprendido suficientemente; las dificultades y carencias enfrentadas, las que se superaron y las que aún están presentes; y las modificaciones y ajustes que el proceso mismo requiere.

- Evaluación que permite ajustar la marcha a partir del reconocimiento de lo que ha sido y lo que se quiere que sea.

- Aprendizaje cuya razón fundamental sea el desarrollo mental y cultural del estudiante y con él, el logro de las capacidades de trabajo intelectual y físico que le permitan comprender y modificar su entorno social, técnico y científico donde le toque actuar y contribuir a su transformación, con miras a su realización personal y social.

\section{Parte II}

La perspectiva de pensar el sistema escolar y en particular la educación superior como un espacio centrado en el aprendizaje genera problemas de diversa complejidad, respecto de los caminos a seguir para concretar el supuesto teórico referenciado. Pero a la vez, visualiza expectativas esperanzadoras de lograr a partir de la exploración y la experimentación, el diseño de planes que impacten en profundo al sistema escolar y canalicen adecuadamente las expectativas sociales sobre su función.

Con el objeto de aproximar la noción de ecuación desde la perspectiva del aprendizaje, se propone un estudio de las relaciones entre diversos aspectos conexos con la noción. Aspectos como el disciplinar, el entorno, el epistemológico entre otros se consideran y examinan con miras a estructurar una red de análisis didáctico que posibilite identificar formas de escolarizar la noción. La complejidad de la red exige la construcción paulatina de segmentos que se centres en nodos. 
Como nodo de la red, se considera un punto de donde salen y a donde confluyen elementos a considerar en la aproximación escolarizada del concepto de ecuación. Dicho nodo puede estar constituido por pre-requisitos del concepto (a su vez pueden ser conceptos), elementos del entorno, conocimientos anteriores vinculados con el concepto (presaberes que no alcanzan a ser nociones), acciones cotidianas que expresan el concepto, expresiones del lenguaje, experiencias del individuo.

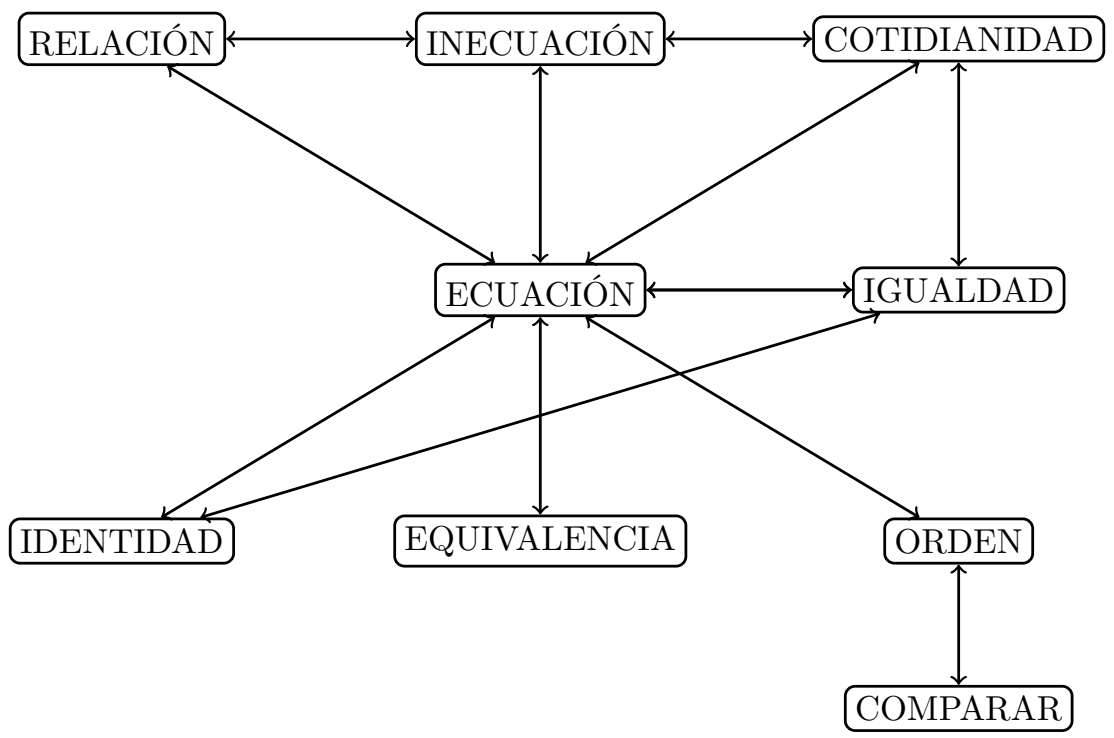

En este caso el segmento de la red permite una lectura de las relaciones de la cotidianidad con otros aspectos. Desde la cotidianidad es posible caracterizar el contexto donde accionan experiencias vinculadas con las ecuaciones cuando se identifican situaciones o problemas que informalmente usan elementos del concepto. El propósito de la identificación de la situación cotidiana es enunciarla y analizarla desde la aritmética informal del individuo (en el caso de las ecuaciones) para determinar, cómo se comprende la situación problema y en qué medida se es capaz de relacionar datos conocidos y desconocidos del mismo. Una mirada al manejo que se da del problema posibilita por parte del maestro determinar cómo piensa el estudiante en el camino de la solución de la problemática vinculada con el concepto; de esta manera posibilitará la identificación y diseño de actividades que permitan acceder a un nivel de mayor comprensión sobre el accionar del concepto.

El acceder a un mayor nivel de comprensión está vinculado con el hecho que en las matemáticas cada elaboración del individuo tiene niveles propios de abstracción y generalización, y por tanto se requieren formas de pensar diferentes que posibiliten saltos cualitativos hacia niveles de mayor abstracción y generalización. En este sentido, la escuela poco tiene en cuenta la cotidianidad del individuo o sus experiencias anteriores e incluso desconoce las experiencias históricas precedentes a la escritura, con lo que refuerza la visión instrumental 
del concepto en consideración (en este caso las ecuaciones) y el carácter idealista de la matemática.

Admitir en el individuo un manejo de elementos relacionados con un concepto que a la vez sea mecánico y comprensivo, es decir, saber de qué se habla y "ser consciente" de lo que se dice, requiere el esfuerzo de comprender (por parte del maestro y del sistema escolar) que la cotidianidad de cada individuo es diferente y por tanto le da carácter particular a la red conceptual.

El maestro como regulador de los procesos del aula debe mantener dentro de los propósitos de su acción docente, la identificación de metas en cuanto a aproximación al concepto que se aborda en el aula y sobre todo debe pensar cómo adelantarse a las posibilidades del estudiante. Esto requiere una posición comprometida con el conocimiento, en oposición a un falso constructivismo fundado en un dejar "hacer cualquier cosa" al estudiante.

Al realizar una mirada (desde la experiencia docente) sobre los procedimientos que realiza un estudiante cuando trata de resolver una ecuación, en general, se aprecia: que si hay un interés por efectuar la tarea, ésta se basa esencialmente en el uso memorístico del algoritmo y no hay un ejercicio de reflexión y elaboración sobre el proceso que subyace al procedimiento. De hecho los procedimientos están en relación directa con las experiencias escolares mediadas por los hábitos, costumbres, formas de actuar y de pensar en tal entorno. Detrás de estos procedimientos se encuentran carencias, las cuales es necesario caracterizar con el objeto de diseñar actividades que ayuden a desarrollar el pensamiento del estudiante en torno a los elementos de las ecuaciones y por consiguiente en los procesos de solución.

Algunas preguntas que acercan la identificación de carencias en el trabajo con ecuaciones son: ¿qué es una ecuación? ¿A qué se refieren las variables de una ecuación? ¿En qué conjuntos numéricos trabajamos las ecuaciones? En este mismo sentido es preciso una exploración de nociones vinculadas a la noción de ecuación, tales como: identidad, igualdad, equivalencia, valor numérico.

El proceso de solución de ecuaciones es uno de los aspectos centrales de la noción de ecuación y tiene vinculaciones profundas con la caracterización de los elementos de los conjuntos numéricos y con el estudio detallado de significaciones en cada uno de ellos de las operaciones y sus propiedades. La situación de uso del signo menos (-) en el proceso de solución debe caracterizarse en cada conjunto numérico donde se van a trabajar las ecuaciones. Por ejemplo en los naturales, el uso del signo no presenta mayores problemas pues significa disminución desde el punto de vista de operador. Al considerar el uso del signo (-) en los enteros, se presenta un problema distinto en cuanto que se conserva el significado que tiene en los naturales y se amplía al uso en cantidades referidas a situaciones contrarias.

Aún más, al considerar el uso del signo menos (-) en otros conjuntos numéricos, se presenta una situación particular en la expresión $2^{-1}$ donde el significado es un acuerdo de notación, diferente a los dos usos antes mencionados y próximo al trabajo con fracciones equivalentes y a propiedades de la potenciación.

$$
1=1^{0}=2^{1-1}=\frac{5}{5}=5 \cdot \frac{1}{5}=5 \cdot 5^{-1}
$$


Por tanto aproximar al estudiante, a tareas relacionadas con la solución de ecuaciones, requiere identificar conocimientos anteriores vinculados con el proceso, con objeto de posibilitar la construcción de explicaciones de carácter matemático en los procedimientos de solución de ecuaciones.

Un aspecto a considerar en el análisis didáctico de las ecuaciones, y en particular el relacionado con el procedimiento de resolución, es el histórico, entendido como el proceso de examinar las realizaciones de culturas primeras de la humanidad en torno al objeto de estudio.

El propósito de la mirada es tratar de encontrar las múltiples experiencias repetitivas de la cotidianidad que posibilitaron la elaboración de las "ideas" próximas a las ecuaciones y a su proceso de solución. Las referencias históricas respecto de las ecuaciones registran elaboraciones de los egipcios en torno las ecuaciones.

En particular se encuentra la solución de la ecuación $x+\frac{1}{7} x=19$.

Los egipcios trabajaron con base en fracciones unitarias, vinculadas posiblemente a la cotidianidad del individuo y a problemas que se plantea desde ésta. Es esta la explicación del antropólogo Frank Hernández quien considera que ha debido ser "más" necesario la acción de "partir por mitades" que la de duplicar, relacionada directamente con la noción de equilibrio, fundamental como visión de mundo en las culturas primeras de la humanidad. Concretamente el equilibrio se puede evidenciar en acciones como poder transportar agua en dos recipientes unidos por un palo y cargados a hombros del individuo, de forma que se garantice "comodidad y efectividad" en dicho transporte. Luego aquí se intuye el significado de "un medio" y se puede intentar una elaboración que en principio tenga relación con el hecho de partir por mitades.

Para el caso de la ecuación planteada veamos una posible explicación de la solución de la ecuación en términos del paso de "uno" o "un montón", a "varias veces uno mismo" o "varias veces el mismo montón". Si se asume que se tenía una conciencia de la relación "parte - todo" donde se identifica como $x$ un montón desconocido, es posible asumir también que se poseía la intuición de que al partir en 7 partes iguales el montón desconocido nombrado como $x$, y juntar de nuevo las siete partes, se obtiene el montón original.

Una posible interpretación de la ecuación en términos "prácticos" apunta a conseguir una expresión para un montón desconocido, de tal forma que 8 "veces" un séptimo del montón equivalga a 19. En término de cantidades discretas es posible el siguiente esquema y el correspondiente procedimiento.

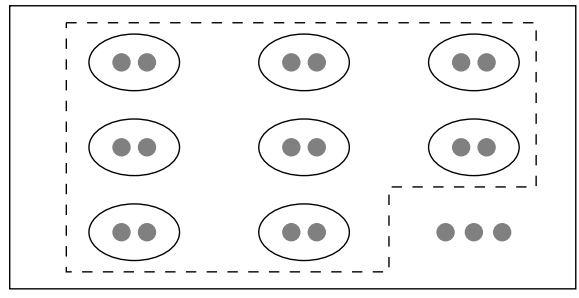

- El montón de 19 objetos debe ser partido en 8 partes iguales. Cada una de estas partes iguales equivaldrá a $\frac{1}{7}$ del montón desconocido nombrado como $x$.

- Se observa que $\frac{1}{7}$ del montón desconocido equivaldrá a 2 más una parte de los tres objetos restantes. 

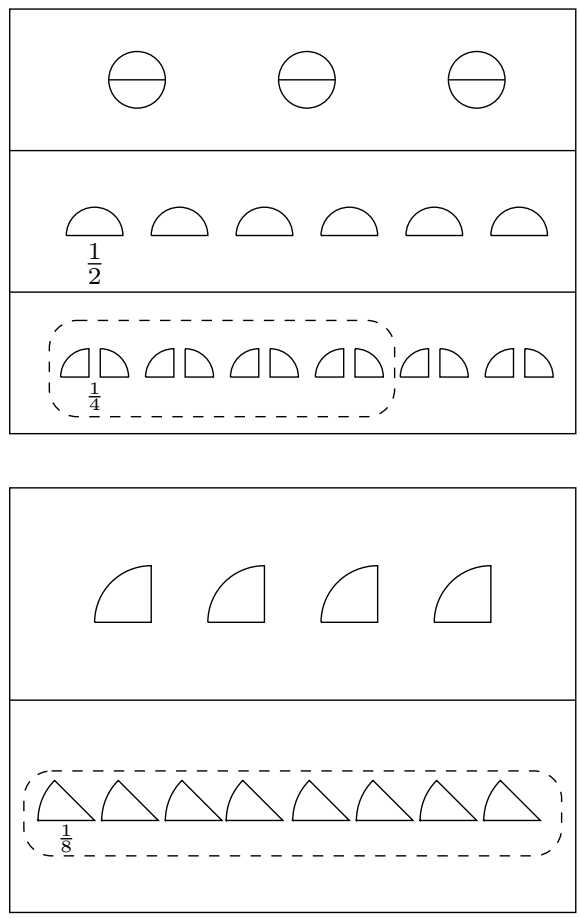

- Al partir los tres objetos restantes en mitades no se alcanza el propósito de partir en 8 partes iguales, así que estas mitades se parten a su vez en mitades.

- Ahora se obtienen 8 partes iguales y cada una equivale a un cuarto de uno de los 19 objetos.

- Se aprecia que quedan 4 partes a repartir de nuevo.

- Estas cuatro partes restantes se parten por mitades obteniéndose 8 partes iguales, cada una de las cuales equivale a $\frac{1}{8}$ de uno de los 19 objetos partidos.

- Por lo tanto $\frac{1}{8}$ del montón buscado equivale a $2+\frac{1}{4}+\frac{1}{8}$.

- En consecuencia el montón buscado es 7 veces $2+\frac{1}{4}+\frac{1}{8}$.

El procedimiento ilustrado es un intento de explicar los procesos posiblemente abordados por una cultura que carecía del símbolo de fracción, pero que evidentemente hacía de la elaboración y la reflexión parte de su cotidianidad con objeto de solucionar inquietudes relacionadas con acciones repetitivas que llevan a interrogantes o a planteamiento de problemas.

Es claro que aquí hay una consideración sobre "lo que se conoce" como medio de elaborar "ideas" y aproximarse a la construcción del "nuevo" objeto, camino que sin duda hace de la explicación matemática el elemento que soporta la justificación del procedimiento que soluciona el problema planteado. Para la universidad queda el cuestionamiento respecto de las formas en que se considera el conocimiento anterior de los estudiantes como elemento que ayuda a aproximarlos a "nuevos" conocimientos y lograr un desarrollo de pensamiento que posibilite acceder a niveles propios de abstracción y generalización, necesarios en diferentes fases de la formación.

La intuición de "partir" por mitades, mitades de mitades, etc. también posibilita un intento de solución de algunas ecuaciones (aquellas donde son posibles particiones referidas) en un continuo como el de los números reales y su representación en la recta numérica. La solución de la ecuación $\frac{4}{3} x=2$ es posible plantearla en estos términos:

Se busca un número desconocido $x$, tal que 4 veces un tercio del número $x$ equivalga a 2. Gráficamente se puede efectuar así: 


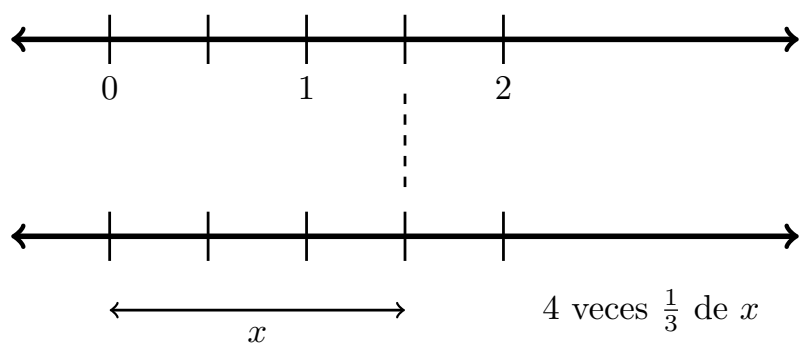

En el gráfico se aprecia entonces que $x=\frac{3}{2}$. Es factible explorar también un proceso de solución de ecuaciones del tipo $a x+b=c$, con $a, b, c$ números tales que $a \neq 0$ de la siguiente forma:

$$
\begin{aligned}
x & =3 \\
x+5 & =3+5 \quad \text { Acción: Agregar } 5 \\
x+5 & =8 \\
2(x+5) & =2(8) \quad \text { Acción: Duplicar (tomar dos veces) las cantidades }
\end{aligned}
$$

\section{Conclusiones}

El sistema escolar y sus componentes deben repensar sus responsabilidades y compromisos frente al aprendizaje. La historia de la evolución de la humanidad es evidencia de los logros alcanzados, cuando el aprendizaje ha sido el elemento central de los proceso de educación y formación del individuo.

El aprendizaje de nociones y conceptos en matemáticas, para estudiantes que acceden a la Universidad, plantea la identificación de elementos que contribuyan bien a la construcción de nuevas nociones y conceptos o bien a destrabar procesos de pensamiento con objeto de re-elaborar nociones y conceptos supuestamente "ya vistos" en la educación básica.

El aprendizaje de nociones y conceptos en matemáticas, cuestiona al docente en cuanto a los soportes de su práctica docente y lo conmina a incorporar a su bagaje intelectual elementos del proceso de investigación como soporte de su intervención pedagógica. Al respecto es preciso revelar y consolidar posiciones respecto a la naturaleza del conocimiento, la importancia del estudio de la disciplina (desde miradas históricas, de evolución del conocimiento y de desarrollos propios de ella) en el progreso de sus visiones sobre el acto educativo y sobre la construcción de su rol en la perspectiva del aprendizaje.

Los recursos del sistema escolar, y en particular los relacionados con las actividades en el aula, deben ser objeto de indagación, reflexión y transformación para alcanzar logros coherentes con los propósitos de una educación matemática desde la perspectiva del aprendizaje como proceso de construcción.

\section{Referencias}

1. Barón E. A., Zamora H. E.: Estrategias para contribuir al aprendizaje comprensivo de nociones y conceptos en Matemáticas, Informe Técnico Final, Politécnico Grancolombiano, Institución Universitaria. (2003) 
2. Bell, A.: Approaches to algebra. Perspective for research and teaching. Kuwer Academic Publishers. Dordreh, The Netherlands. (1996)

3. Kline, M.: El pensamiento matemático de la antiguedad a nuestros días. Alianza Editorial. Madrid. (1992)

4. Mardones, J.M.: Filosofía de las ciencias humanas y sociales. Materiales para una fundamentación científica. Editorial Anthropos. Barcelona. (1991)

5. Ortíz, M.: Iniciación a la aritmética. Una propuesta de formación de maestros desde la perspectiva del aprendizaje. Tesis de doctorado. Centro de estudios avanzados del Instituto Politécnico Nacional de México. D.F. Departamento de Matemática Educativa. México. D.F. (1995)

6. Ursini, S., Trigueros, M.: Understanding of different uses of variable. Twenty-First Annual Conference of PME. Lathi, Finlandia. (1997)

7. Vasco C.E.: Pedagogías para la comprensión en las disciplinas académicas, Unipluri/versidad, Vol. 4, No. 1. (2004) 